\title{
The Mysteries of the Highest Energy Cosmic Rays
}

\author{
S. Kovesi-Domokos ${ }^{\mathrm{a} *}$ and G. Domokos ${ }^{\mathrm{a} \dagger}$ \\ ${ }^{a}$ Department of Physics and Astronomy \\ The Johns Hopkins University, Baltimore, MD
}

We give a summary of the theoretical attempts to make sense of the observational data concerning the highest energy cosmic rays, $E>3 \times 10^{19} \mathrm{eV}$.

\section{Preamble}

It was about forty years ago that Linsley [1] presented an incredulous physics community with the $\sim 10^{20} \mathrm{eV}$ event observed at the Volcano Ranch array. Today ground arrays and fluorescence detectors have collected over a hundred events with energies above $3 \times 10^{19} \mathrm{eV}$, and a dozen or so at or above $10^{20} \mathrm{eV}$. It is worth remembering that the $\sim 2 \mathrm{TeV} \mathrm{CM}$ energies attained at the Tevatron are at the knee of the cosmic ray spectrum. LHC will operate at an equivalent fixed target energy falling approximately halfway between the knee and the ankle, around the so-called "second knee" region. Thus, above the second knee, the first interaction in an extensive air shower involves physics untested by the well-controlled experimental environments of accelerators.

For many years, the appearance of the GZK feature [2] at the highest energies (i.e. above $\left.2 \times 10^{19} \mathrm{eV}\right)$ was taken for granted. The processes, $p+\gamma_{\mathrm{CMB}} \rightarrow \pi+X$, and, of lesser importance, $p+\gamma_{\mathrm{CMB}} \rightarrow e^{+} e^{-}+X$, are well-studied low energy processes in the CM. A proton of energy $10^{20} \mathrm{eV}$ or above has a mean free path of $7-8 \mathrm{Mpc}$ in the cosmic microwave background (CMB) radiation. With each interaction event, the proton loses $20-25 \%$ of its energy; after only a few interactions its energy drops under the threshold of the pion photoproduction process. The expected precipitous drop in the incoming

\footnotetext{
*Speaker. e-mail: skd@jhu.edu Invited talk given at XIII ISVHECRI, Pylos, Greece.

${ }^{\dagger}$ e-mail: Gabor.Domokos@jhu.edu
}

cosmic ray flux at $5 \times 10^{19}-8 \times 10^{19} \mathrm{eV}(\mathrm{GZK}$ cutoff) and the pileup [3] (a bump in the spectrum) just before the drop" ${ }^{3}$ are called "the GZK feature". The GZK sphere approximately defines the cosmic neighborhood of sources from which we expect the arrival of extreme high energy cosmic rays $\left(E \approx 10^{20} \mathrm{eV}\right.$ and up). We usually put this particle horizon at 50-70 Mpc. However, the GZK radius could be considerably less as demonstrated by Stanev et al. 4]. Scattering in extragalactic random magnetic fields makes the real distance travelled by the particle longer than the source-Earth distance; more energy loss processes can take place as a result. Heavier nuclei with similar energies also suffer energy loss through photodisintegration on the cosmic microwave and on the intergalactic infrared background radiations 5]. For ${ }^{56} \mathrm{Fe}$, for example, the decrease of the energy loss time is even more abrupt than for protons, though it starts at a somewhat higher energy.

We will summarize the most important, and often controversial, observational data. Then we present the theories formulated to explain certain coherent subsets of the data.

\section{Observational Data}

What follows is a very brief summary of the most important features of the extensive air shower and EGRET data from a theorist's point of view. Only ultra high energy cosmic rays (UHECR) with energies above $3 \times 10^{19} \mathrm{eV}$ are

\footnotetext{
${ }^{3}$ This is a consequence of the pion photoproduction by the
} above threshold protons or nuclei. 
considered.

\subsection{Spectrum}

At and above $10^{20} \mathrm{eV}$, there are 15 events: 11 observed by AGASA [6] and 2 by HiRes [7] and one each by Fly's Eye [8], and Yakutsk $[9]$.

There is a substantial disagreement between AGASA and HiRes at this time. The HiRes data were successfully fitted 11] with a two-component model 10 including primaries with galactic and extragalactic origins. The Fly's Eye data suggest a transition from heavy to light (proton) primaries (at energies from $10^{17}$ to $10^{19} \mathrm{eV}$ ), which can be interpreted as a sign of the passage from galactic to extragalactic sources. The extragalactic source model of Berezinsky et al. [12, which takes into account both uniform source distribution and a maximal energy at $10^{21} \mathrm{eV}$, yields not only the GZK feature but also other details of the spectrum, as a result of considering both energy loss processes. The large number of events above the GZK cutoff (mostly observed by AGASA) remains unexplained by the twocomponent models ${ }^{4}$.

It should be emphasized that even the two largest experiments, AGASA and HiRes, have too few events at the highest energies; the discrepancy between them has low statistical significance as a result [13.

\subsection{Composition}

As mentioned above, fluorescence detectors provide some hints for a $\mathrm{Fe} \rightarrow \mathrm{p}$ transition. However, it is important to note that the mass composition results are different for each of the various methods and model calculations used 14. In addition, the shower energy estimates are sensitive to the assumption about the nature of the primary. To quote A.A. Watson (loc. cit.), "knowledge about the mass of the primary cosmic rays at energies above $10^{17} \mathrm{eV}$ is rudimentary." As far as photon primaries are concerned, it is very

\footnotetext{
${ }^{4}$ Since for both AGASA and HiRes there are systematic errors in the energy assignment, it is difficult to judge how great the disagreement between them actually is.
}

unlikely that the primary of the "gold-plated" $3 \times 10^{20} \mathrm{eV}$ Fly's Eye event was a photon [15]. Around $10^{19} \mathrm{eV}$, the photon flux is less than $40 \%$ of the baryonic component according to both Haverah Park [16] and AGASA [17. By searching for deeply penetrating horizontal air showers, AGASA investigated the UHE neutrino flux for energies $>3 \times 10^{17} \mathrm{eV}$ [18]. No significant enhancement above the expected hadron-induced flux was reported.

\subsection{Directionality}

For the energy range we are interested in, the arrival direction distribution is remarkably isotropic. This could indicate a large number of weak or distant sources. AGASA data show small-scale clustering [19] (4 doublets and one triplet within a separation angle of $2.5^{\circ}$ ) above $4 \times 10^{19} \mathrm{eV}$ (57 events). HiRes in stereo mode above $10^{19} \mathrm{eV}$ (162 events) claims to see no smallscale anistropy in the arrival direction distribution 20. Recently, it was found 21] that the statistical significance here is also very low for both the small scale clustering in the AGASA data set and the apparent discrepancy between AGASA and HiRes.

Nevertheless, despite the paucity of data points, statistical analyses have attempted to discover possible angular correlations between the arrival directions of the extremely high energy cosmic rays (EHECR), and to match these in turn with high energy source canditates. The enticement to carry out an analysis of even the small available sample is obvious. Significant angular correlation in the arrival directions of the EHECR could be the a smoking gun for the existence of discrete compact (repeating) sources. Using the AGASA data of $E>4 \times 10^{19} \mathrm{eV}^{5}$, the presence of clustering can be used to constrain the number of sources in the nearby Universe for proton primaries 22 to be $10^{-5} \mathrm{Mpc}^{-3}$ with large uncertainties. Tinyakov and Tkachev [23] claimed to have identified BL Lacertae objects as sources generating the anisotropies in the

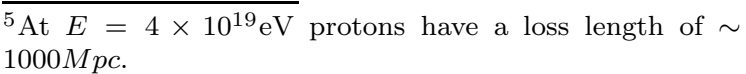


AGASA data. Evans et al. 24 and W. Burgett et al. 25] questioned this conclusion, however ${ }^{6}$.

Most authors working in the field agree that more data from HiRes and from larger experiments like the Southern (and hopefully the Northern) Pierre Auger Observatory, EUSO, the Telescope Array, and OWL are necessary to draw solid conclusions about clustering and the presence or absence of the GZK cutoff discrepancies. Until then the BL Lac alignment is merely suggested by the need for objects accelerating particles to exceptional energies, rather than being a firmly established consequence of the observational data.

\subsection{Extragalactic Diffuse Gamma Rays}

Another piece of important data is the extragalactic diffuse $\gamma$ ray background (EGRB). It is generally believed that unresolved AGN, galaxy clusters, distant gamma ray bursts, etc. are the main sources. However, EGRB would get a significant contribution from the decay of topological objects, relic particles etc., which are used by some authors to explain trans-GZK cosmic ray events. Comparing that contribution to the measured EGRB, one may be able to place upper limits on, or eventually exclude, some of those schemes. Any electromagnetic (EM) energy introduced to the EM background radiation (dominated by $\mathrm{CMB}$ ) produces an EM cascade if the energy is $\gtrsim 10^{15} \mathrm{eV} /(1+z)$ i.e. the pair production "threshold" on the CMB at redshift z. The cascade by pair production and inverse Compton scattering degenerates the injected energy to under $\sim 10^{15} \mathrm{eV}$ in a couple of Mpc and produces a power-law distribution of GeV-range gamma rays.

To obtain the EGRB, one must construct a model of the galactic $\gamma$ ray background, subtract

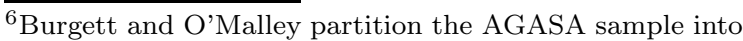
three statistically uncorrelated groups by energy: $E<$ $5 \times 10^{19} \mathrm{eV}, 5 \times 10^{19} \leq E<8 \times 10^{19} \mathrm{eV}$, and $E>$ $8 \times 10^{19} \mathrm{eV}$. They find the middle group to be preferentially aligned with the Supergalactic equatorial plane, while the other two groups are statistically consistent with isotropic distributions.
}

it from the observational data, and assign the remainder to the EGRB. This is a complicated and model-dependent process. The EGRB was first discovered with SAS-II 26 and then confirmed by EGRET [27. A power law energy spectrum $\left(E^{(-(2.1 \pm 0.03))}\right.$ in the range $30 \mathrm{MeV} \lesssim E \lesssim 100$ $\mathrm{GeV}$ ) with no anisotropy was found. Strong et al. 28, in a recent reworking of the EGRET data, substantially improved the determination of the diffuse galactic $\gamma$ ray flux. They conclude that in previous analyses the galactic contribution was underestimated; the EGRB must be revised downward as a result. The new spectrum is not consistent with a power law. One may infer tentatively that the EGRB arises from a number of different physical processes.

At higher energies $(20 \mathrm{TeV} \lesssim E \lesssim 500 \mathrm{TeV})$, an upper limit on the EGRB can be obtained by estimating the ratio of photon-induced to hadroninduced extensive air showers. The GRAPES collaboration 29] obtained an upper limit of about $3 \times 10^{-4}$ for the fraction of photon-induced (muon-poor) air showers.

\section{Theoretical Models}

The presence or absence of the GZK feature determines whether it is enough to rely on conventional astrophysics to explain the spectrum, composition, and directionality encrypted in the data, or if physics beyond the Standard Model is needed 30.

\subsection{Traditional Astrophysics}

These models are also called bottom-up scenarios. At $10^{19} \mathrm{eV}$ and above, the extragalactic component must dominate the energy spectrum. Even if there are some objects (neutron stars, galactic wind, etc) in our galaxy which could possibly accelerate UHECR, it would be impossible for them to produce the observed isotropic distribution. For order of magnitude estimates, the community generally uses Hillas' formula. Whether the acceleration takes place electromagnetically or with the help of diffusive shock acceleration (first order Fermi process), the magnetic field must be strong enough to keep the particles 
in the region of acceleration, thus, approximately,

$$
R_{\text {accelerator }} \gtrsim R_{\text {gyromagnetic }} \approx \frac{E}{Z e B},
$$

where the charge of the accelerated particle is $Z e$. From this,

$$
E_{\text {max }} \approx Z e B R_{\text {accelerator }}
$$

We are looking for a maximal energy of about $10^{21}-10^{22} \mathrm{eV}$. The enormous energy of the primary singles out exceptional astrophysical sources: radio lobes of powerful radio galaxies, jets of active galactic nuclei, gamma ray bursters, and magnetars. There are only a few of these possible accleration sites inside the GZK radius for charged hadrons. (For example, the radio galaxy M87, in the center of the Virgo cluster, was investigated repeatedly as a candidate 31. It could provide EHECR at Earth only if the extragalactic magnetic fields on the way here have some specific configuration.) Consequently, it is unlikely that the GZK cutoff could be completely absent in the spectrum. The accelerators are far beyond the pion photoproduction distance and the random, poorly known, extragalactic magnetic fields may cause deflection of charged particles. In fact, the relevant sources are at cosmological distances. Nevertheless there are small scale anisotropies and correlations with certain sources. These observations indicate that the cosmic traveler may be a stable, neutral particle which interacts very weakly with the cosmic background radiation fields. This particle could be a neutrino.

It is generally accepted that the dominant form of acceleration of cosmic rays at the sources is by first order Fermi acceleration at astrophysical shocks. A recent development in the theory of shock acceleration is the introduction of the back reaction of the accelerated particles to the shock modifying it strongly [32. Among other results the spectrum is claimed to be flatter than in the linear theory; thus, the number of arriving transGZK primaries could be higher.

\subsection{Scenarios Beyond the Standard Model}

\subsubsection{Z-bursts}

The Z-burst model 33 needs only the inclusion of the neutrino mass in the Standard Model (SM) for its attempt to explain the trans-GZK events. Among SM particles only the neutrino can handle cosmic distances without dramatic absorption. The assertion is that extreme energy neutrinos

$$
\begin{aligned}
& E_{\text {res }}=M_{Z}^{2} / 2 m_{\nu}=4 \times 10^{21}\left(m_{\nu}(\mathrm{eV})\right)^{-1} \mathrm{eV} \\
& =4 \times\left(m_{\nu}(\mathrm{eV})\right)^{-1} \mathrm{ZeV}
\end{aligned}
$$

forming $Z^{0}$ bosons resonantly with antineutrinos of the relic $1.9^{\circ} \mathrm{K}$ thermal neutrino background could produce enough protons well inside the GZK radius to account for the EECR. On average a $Z^{0}$ produces $\sim 2$ nucleons, $\sim 20$ photons and $\sim 50$ neutrinos. Although the Z-burst model is very economical, but it is likely to have fatal problems both because of the required energy at the source and because of the extreme energy neutrino flux required to produce enough of the trans-GZK events 34. The new experimental limit by WMAP, $2 \mathrm{dF}$ and SDSS on the active neutrino mass $\left(\sum m_{\nu i} \lesssim 0.72 \mathrm{eV}\right)$ combined with the neutrino oscillation results gives the maximal possible neutrino mass $m_{\nu} \approx 0.24$ $\mathrm{eV}$ [35. This puts the energy of the neutrino for the resonant production at $\sim 10 \mathrm{ZeV}$. If these neutrinos are secondaries of pions, the protons in the source must be accelerated to $\sim 10^{3} \mathrm{ZeV}$. No known astrophysical object is capable of accelerating protons to such high energies [36]. A large enough flux by ordinary astrophysical sources (or by beyond-the Standard Model objects [37]) to produce a substantial part of trans-GZK events would pump so much EM energy into the universe that the EGRET limits would be violated many times over.

An overdensity of neutrinos in a halo of our galaxy or in that of the Local Group could help to reduce the flux requirement. However, these neutrino masses are too small to allow any clustering of relevance to allow the reduction of the 
incoming neutrino flux 38, 39. For a conservative maximal neutrino mass, $m_{\nu} \approx 0.33 \mathrm{eV}$, the neutrino flux required by the Z-burst scenario is already in marginal conflict with the upper limit given by the FORTE 40] experiment and in violation of the GLUE 41] limit. A lower mass makes the problem more severe [39]. This limit on the Z-burst model is valid even when the distant sources emit only neutrinos.

\subsubsection{Strongly Interacting Neutrinos}

The problem of energy loss during the propagation at cosmological distances would not arise if the trans-GZK primaries were neutrinos and interacted strongly at very high energies 42, causing proton-like air showers. Both the energy and flux reqirements are much lower here than in the Z-burst model. In this case the cosmogenic neutrino flux (i.e. secondaries from the pion photoproduction of extreme energy protons on the CMB) could account for the trans-GZK events 43, since all incoming neutrinos produce an air shower. For the protons the injection spectrum is simple, power law. The onset of strong interaction must result in a very rapidly rising cross section; a slow rise would give a large number of horizontal showers, which are not seen by either AGASA or HiRes. For example, in theories of higher than 4 dimensions, Kaluza-Klein graviton exchange gives a power law increase, which is not only too slow, but strong interaction cross sections cannot be reached until energies $\approx 10^{20} \mathrm{eV}$ [4].

The following two schemes also assume some higher dimensional (string inspired) theory. The neutrino-nucleon cross section begins to grow at a certain energy, in essence due to the unification of all interactions at a scale of the order of a few tens of $\mathrm{TeV}$ or a few $\mathrm{TeV}$ in the CMS, producing lepto-quark resonances [4], mini black holes 46] or "p-branes" [4]. The asymptotic $(s \rightarrow \infty)$ equivalence of the resonance and mini black hole schemes was recently demonstrated [4]. The onset of the new interaction is very different. In the "semiclassical black hole" picture, it is assumed that the cross section is proportional to the square of the higher dimensional $(d=10$ ?) Schwarzschild radius. Combined with the assumption that $m_{\mathrm{bh}} \propto \sqrt{s}$, it leads to an unacceptably slow rise of the cross section. The published data on near horizontal showers by AGASA and Fly's Eye exclude that. By contrast, with a string scale of the order $70 \mathrm{TeV}$ one is far below the semiclassical region 49]. The growth of the cross section is governed by the exponentially increasing level density in the transition region (and only in the transition region) from the SM to the string regime. In this scheme few near horizontal showers are produced, as a result.

Another possibility, suggested to achieve strong cross sections, is the inclusion of instanton induced processes. There is a detailed discussion and further references on the instanton enhanced cross section in 43 .

It is remarkable that the cosmogenic neutrino beam, together with a strongly interacting neutrino with sudden onset of large cross section could give a solution of the trans-GZK events. This is accomplished without the need to assume exotic astrophysical scenarios. There is no obvious contradiction with data obtained from FORTE and GLUE, either.

\subsubsection{Top-down models}

Very heavy topological defects (TD) could solve both the problem of the long trip for protons in the CMB and the difficulty to accelerate particles to extreme energies [37. In the early universe at the time of phase transition from the GUT scale $\left(M \geq 10^{14} \mathrm{GeV}\right)$ TD (monopoles, cosmic strings, necklaces, textures etc.) could be formed in localized regions where mass-energy is trapped. In the decay (or annihilation) of these superheavy extragalactic relics, "X-particles" are formed which decay trough QCD fragmentation jets consisting mostly of photons, neutrinos and $\sim 5 \%$ nucleons. These nucleons provide the trans-GZK events. However, a large fraction of the energy is injected into the extragalactic space via $\gamma$-s with $E>10^{15} \mathrm{eV}$. Thus, if the TD can 
generate the flux of EECR, then the EGRET limit (see 2.4) is always exceeded [50].

Another type of top-down scheme uses the possible existence of superheavy dark matter particles, which could have been created at the end of inflation in the early Universe. Their decay or annihilition could also produce the trans-GZK spectrum via QCD fragmentation [51]. As cold dark matter, they could have an overdensity in the halo of our galaxy by a factor of $10^{4}$. Their mass must be $>10^{12} \mathrm{GeV}$ and the extreme energy protons will reach the detectors since they are produced closeby. However, there is anisotropy in the arrival direction because of our position in the galaxy. In addition, the fragmentation process produces about a factor of 2 more ultra high energy photons than protons. As it was discussed in Section 2.2, the experimental photon fraction is certainly $<1$.

\subsubsection{Stable Light Neutral Particles}

The idea of light, supersymmetric, stable or long-lived hadrons instead of nucleons as UHECR was entertained for some time. A mass larger than the mass of the nucleon $(\approx 2 \mathrm{GeV})$ would increase the threshold energy of the $\pi$ photoproduction and push the GZK cutoff to higher energies.

One hypothetical particle in this class [52] required the existence of a light gluino. Precision measurements at LEP and SLC closed the socalled "light gluino window" 53. Shadrons with a light sbottom were investigated as well [54, motivated by the preliminary result from the Tevatron indicating an overproduction of $b \bar{b}$. Futher data taking, however, did not confirm the effect 55.

The possibility of axion-photon mixing was considered in Ref. [56]. The hypothesis was that the trans-GZK showers were started by regenerated photons, while the axions were the long distance travelers. One may object, however, that according to present observational data the transGZK showers look hadronic and not photon- induced.

\subsubsection{Lorentz Invariance Violation}

The idea of small violation of Lorentz invariance is not new [57]. The idea was reconsidered again as a possible explanation for the lack of the GZK cutoff in the AGASA and Fly's Eye data 58 as well as a putative absence of high energy photon absorption on the infrared (IR) background $[59]^{7}$. A review of the various schemes can be found in ref. 60. There is no firmly established scheme of violating Lorentz invariance at present. The most popular scenario invoked in explaining the existence of trans-GZK cosmic ray events follows the paradigm laid out in Ref. [58]. According to that scheme, Lorentz invariance is violated by assigning different maximal speeds to different particle species (for instance, to electrons and photons), none of them or some of them being equal to what is known to be the speed of light in vacuo.

In this manner, the rate of pair production by photons and the rate of photoproduction by protons can be suppressed by invoking a rather small amount of Lorentz invariance violation. Conversely, the absence of trans-GZK flux in future experiments could place severe constraints on Lorentz invariance violation. It is important to point out that the same set of assumptions leads to some rather dramatic changes in the development of cascade showers, see ref. 61. A violation of Lorentz invariance as an explanation of trans-GZK event cannot be ruled out at present.

\section{Conclusion}

A plethora of particle physics models was created to explain the trans-GZK events, which are anomalous according to expectations based on astrophysics and on SM. Z-bursts, TD seem to be ruled out already to a large extent by the extragalactic diffuse $\gamma$-ray background measurements.

\footnotetext{
${ }^{7}$ We were informed by Michael Fall (private communication) that the uncertainties in our knowledge of the IR background are considerable.
} 
Superheavy dark matter is fatally wounded according to the available observational data on the ratio of UHECR photons to protons and on the lack of directional asymmetry. Strongly interacting neutrinos and Lorentz invariance violation are still alive. The nature and origin of EHECR is still an enigma. If the observational data from the next generation of detectors support the existence of trans-GZK events then the next great leap for particle physics could come from nonaccelerator experimental physics. It is evident that our physics and astrophysics ideas can truly be changed by the results coming from the continuing and the newly commissioned detectors: HiRes, Telescope Array, P. Auger Observatory, EUSO, OWL, AMANDA, ICECUBE, NESTOR, NEMO, ANTARES, HESS, VERITAS, MAGIC, GLUE, FORTE etc.

\section{Acknowledgements}

We would like to thank V. Berezinsky and W. Burgett for valuable discussions. Special thanks are due to the organizers of XIII ISVHECRI, in particular to Leo Resvanis for hosting such an informative and stimulating meeting. We further thank the organizers for financial support.

\section{REFERENCES}

1. J.Linsley, Phys.Rev.Lett. 10, 146 (1963).

2. K.Greisen, Phys. Rev. Lett. 16, 748 (1966); G.T. Zatsepin, and V.A. Kuzmin Pisma Zh. Eksp. Theor. Fiz. 611028 (1966).

3. C.T. Hill, D.N. Schramm, Phys. Rev. D31, 564 (1986); V.S. Berezinsky, S.I. Grigorieva Astron. Astrophys. 199, 1 (1988).

4. T. Stanev, R. Engel, A. Mücke, R.J. Protheroe, and J.P. Rachen Phys. Rev. D62, 093005 (2000); also A. Achterberg et al. astro-ph/9907060

5. F.W. Stecker, M.H. Salamon ApJ. 512, 521526 (1999); T. Yamamoto, et al., Astropart. Phys. 20, 405 (2004).

6. M. Takeda et al., Astropart. Phys. 19, 447 (2003)

7. T. Abu-Zayyad et al., astro-ph/0208301. (submitted to Astropart. Phys.)
8. D.J. Bird, et al., Phys. Rev. Lett. 71, 3401 (1993).

9. N.N. Efimov et al., in Astrophysical Aspects of the Most Energetic Cosmic Rays, eds. M.Nagano and F.Takahara (World Scientific, 1991) p20.

10. J.N. Bahcall and E. Waxman, Phys. Lett. B556, 1 (2003).

11. D.R. Bergman, et al.,Proc. of 28th ICRC (Tsukuba), 683 (2003); J.N. Matthews (for the collaboration) Acta Phys. Polon. 351863 (2004).

12. V. Berezinsky, A.Z. Gazizov, and S.I.Grigorieva, hep-ph/0204357 and also astro-ph/0210095

13. D. De Marco, et al., Astropart. Phys. 20, 53 (2003).

14. A.A. Watson, The mass composition of cosmic rays above $10^{17} \mathrm{eV}$; these Proceedings.

15. F. Halzen, R.A. Vazquez, T. Stanev, H.P. Vankov Astropart. Phys. 3, 151 (1995); also an analysis of the $3 \times 10^{20} \mathrm{eV}$ event was given by M. Risse, et al., Astropart. Phys. 21, 479 (2004).

16. M. Ave, et al., Phys. Rev. Letters 85, 2244 (2000).

17. K. Shinozaki, et al., Astrophys. J. 571, L117 (2002).

18. S. Yoshida, Proc.of 27th ICRC (Hamburg) 3, 1142 (2001).

19. M. Takeda, et al., Proc. of 27th ICRC (Hamburg), 341 (2001).

20. C.B. Finley and S. Westerhoff, for the HiRes collaboration, Proc. of 28th ICRC (Tsukuba), 433 (2003).

21. C. B. Finley, S. Westerhoff, Astropart.Phys. 21, 359 (2004); H. Yoshiguchi, et al., astro-ph/0404411.

22. P. Blasi, and D. De Marco, Astropart. Phys. 20, 559 (2004); M. Kachelriess, and D.V. Semikoz, astro-ph/0405258

23. P.G. Tinyakov, I.I. Tkachev, Pisma Zh. ksp. Teor. Fiz. 74, 3 (2001) ( JETP Lett. 74, 1 (2001)) and Phys.Rev. D69,128301 (2004).

24. N.W. Evans, F. Ferrer, Subir Sarkar, Phys.Rev. D67, 103005 (2003); ibid. D69,128302,(2004).

25. W.S. Burgett and M.R. O'Malley, Phys. Rev. 
D67, 092002 (2003).

26. D.J. Thompson and C.E. Fichtel, Astron. Astrophys. 109, 352 (1982).

27. P. Sreekumar, et al., Astrophys. J. 494, 523 (1998).

28. A.W. Strong, I.V. Moskalenko and O. Reimer, Astrophys. J. 613, 956 (2004).

29. Y. Hayashi et al in Proc. $28^{\text {th }}$ ICRC, Tsukuba, Japan (2003).

30. There are review papers (with no page limitation), in which more detailed discussions of these questions can be found, for example, M. Nagano and A.A. Watson, Rev. Mod. Phys, 72, 389 (2000); R.J. Protheroe and R.W. Clay, Publ. Astron. Soc. Pac. 21, 1 (2004); F.W. Stecker, astro-ph/0407311 articles in Physics and Astrophysics of UHECR, Eds. M. Lemoine, G. Sigl (Springer 2001).

31. R.J.Protheroe, et al., Astropart. Phys. 9, 559 (2003), and references quoted there.

32. F.C. Jones and D.C. Ellison, Space Sci. Rev. 58, 259 (1991); P. Blasi, astro-ph/0411056

33. T.J. Weiler, Astropart. Phys. 11, 303 (1999); D. Fargion, et al. Astrophys. J. 517 (1999).

34. G. Domokos and S.Kovesi-Domokos, in IEC-HEP, JHEP Conf.Proc. (2001); hep-ph/0107095

35. G. Bhattacharyya, et al., Phys. Lett. B564, 175 (2003); S.W. Allen, et al., Mon. Not. Roy. Astr. Soc. 346, 593 (2003).

36. O.E. Kalashev, et al., Phys. Rev. D65, 103003 (2002); D.S. Gorbunov, et al., Astropart. Phys. 18, 463 (2003).

37. P. Bhattacharjee and G. Sigl, Phys. Reports 327, 109 (2000).

38. Q. Shafi and F.W. Stecker, Phys. Rev. Lett. 53, 1292 (1984); S. Singh, and C.-P. Ma, Phys. Rev. D67, 023506 (2003).

39. D.V. Semikoz, G. Sigl, JCAP 0404, 003 (2004).

40. N.G. Lehtinen, et al., Phys. Rev. D69,013008 (2004).

41. P.W. Gorham etal., Phys. Rev. Lett. 93, 041101 (2004).

42. This was first suggested by V.S. Berezinsky and G.T. Zatsepin, Phys. Lett. B28, 423 (1969).
43. Z. Fodor, et al., Phys. Lett. B561,191 (2003), and Z. Fodor, et al., contribution to the 10th M. Grossmann Meeting, hep-ph/0402102

44. For a recent review see L. Anchordoqui, et al., Int. J. Mod. Phys. A18, 2229 (2003).

45. G. Domokos and S. Kovesi-Domokos, Phys.Rev.Lett.82,1366 (1999).

46. L.A. Anchordoqui, J.L. Feng, H.Goldberg, A.D. Shapere, Phys.Rev. D65,124027 (2002).

47. E.J. Ahn, et al., Phys. Lett. B 551, 1 (2003).

48. G. Domokos and S. Kovesi-Domokos, hep-ph/0307099 and G. Domokos and S. Kovesi-Domokos, in these proceedings.

49. W.S. Burgett, et al. hep-ph/0209162 S. Kovesi-Domokos, G. Domokos, 26th Johns Hopkins Workshop (Heidelberg, Germany), JHEP Conference Proceedings.

50. R.J. Protheroe and T. Stanev, Phys. Rev. Lett. 77, 3708 (1996).

51. V.S. Berezinsky, et al., Phys. Rev. Lett. 79, 4302 (1997); M. Birkel and S. Sarkar, Astropart. Phys. 9,297 (1998); K. Benakli, et al., Phys. Rev. D59, 047301 (1999); D.J.H.Chung, et al., Phys. Rev. Lett. 81, 4048 (1998); N. Rubin, M.Phil.Thesis, U. of Cambridge (1999); Z. Fodor and S.D. Katz, Phys. Rev. Lett. 86, 3224 (2001); S. Sarkar and R. Toldra, Nucl.Phys. B621, 495 (2002); P. Blasi, et al., Astropart. Phys. 18, 57 (2002).

52. G.R. Farrar,Phys. Rev. Lett. 76, 4111 (1996); D.J.H. Chung, et al., Phys. Rev., D57, 4606 (1998); V.S. Berezisky, et al., Phys. Rev. D65, 083004 (2002).

53. P. Janot, Phys. Lett. B564, 183 (2003).

54. M. Kachelriess, et al., Phys. Rev. D68,043005 (2003).

55. M. Bishai, private communication.

56. C. Csaki, et al., JCAP 0305, 005 (2003).

57. H. Sato and T. Tati, Prog. Theor. Phys. 47, 1788 (1972); S.M. Carroll, et al., Phys. Rev. D41, 1231 (1990).

58. S. Coleman and S. Glashow, Phys. Rev. D59, 116008 (1999) and references cited there; L.Gonzalez-Mestres physics/9706032 in Proc. $25^{\text {th }}$ ICRC. G. Amelino-Camelia and T. Piran, Phys. Rev. D64, 036005 (2001).

59. R.J. Protheroe and H. Meyer, Phys. Lett. B493, 1 (2000). 
60. F.W. Stecker, astro-ph/0409731 F.W. Stecker and S.T. Scully, astro-ph/0412495

61. H. Vankov and T. Stanev, Phys. Lett. B538, 251 (2002). 